\title{
COMUNICAÇÃO
}

\section{PARASITÓIDES DE Fannia pusio (Wiedemann, 1830) (Diptera: Fanniidae) COLETADOS EM CALDAS NOVAS, GOIÁS, BRASIL}

\author{
Parasitoids of Fannia pusio (Wiedemann, 1830) (Diptera: Fanniidae) collected in Caldas Novas, \\ Goiás, Brasil
}

\author{
Carlos Henrique Marchiori ${ }^{1}$, Otacílio Moreira Silva Filho ${ }^{1}$, Francilene Cardoso Alves Fortes ${ }^{2}$, \\ Rélia Rodrigues Brunes ${ }^{2}$, Rauer Ferreira Borges ${ }^{2}$, Patricia Luzia Pereira Gonçalves ${ }^{2}$, Juliana Fischer Laurindo ${ }^{2}$
}

\begin{abstract}
RESUMO
Com este estudo, objetivou-se verificar as espécies de insetos parasitando Fannia pusio (Wiedemann, 1830) (Diptera: Fanniidae) em Caldas Novas, Goiás, de agosto de 2003 a maio de 2004, empregando como atrativo de alimentação iscas formadas por fezes humanas, fígado bovino e peixe, com pupas sendo isoladas pelo método da flutuação, em água e individualizadas em cápsulas de gelatina até a emergência das moscas e/ou dos seus parasitóides. As porcentagens de parasitismo apresentada por Pachycrepoideus vindemmiae (Rondani, 1875) (Hymenoptera: Pteromalidae), Spalangia nigra Latrielle, 1805 (Hymenoptera: Pteromalidae) Paraganaspis egeria Díaz, Gallardo \& Walsh., 1996 (Hymenoptera: Figitidae) e Spalangia drosophilae Ashmead, 1887 (Hymenoptera: Pteromalidae) foram de 4,3, 1,5 0,9 e 0,6\%, respectivamente. A porcentagem total de parasitismo observada foi de 7,4\%. Relata-se a primeira ocorrência de S. nigra em pupas de F. pusio no Brasil.
\end{abstract}

Termos para indexação: Diptera, Hymenoptera, inimigo natural, moscas, armadilhas.

\begin{abstract}
The aim of this study was to report the insect species parasitizing Fannia pusio (Wiedemann, 1830) (Diptera: Fanniidae), in Caldas Novas, State of Goiás, between August 2003 and May 2004. Flies were attracted to baits consisting of human feces, bovine liver and fish, with the pupae being isolated by the flotation method, in water, and individually placed in gelatin capsules until the emergence of the flies and/or their parasitoids. The parasitism percentages presented by Pachycrepoideus vindemmiae (Rondani, 1875) (Hymenoptera: Pteromalidae), Spalangia nigra Latrielle, 1805 (Hymenoptera: Pteromalidae), Paraganaspis egeria Díaz, Gallardo \& Walsh, 1996 (Hymenoptera: Figitidae) and Spalangia drosophilae Ashmead, 1887 (Hymenoptera: Pteromalidae) were $4.3,1.5,0.9$ and $0.6 \%$, respectively. The total percentage of parasitism was $7.4 \%$. This study reports the first occurrence of $S$. nigra in pupae of $F$. pusio in Brazil.
\end{abstract}

Index terms: Diptera, Hymenoptera, natural enemy, flies, traps.

(Recebido para publicação em 21 de outubro de 2004 e aprovado em 13 de maio de 2005)

Os dípteros muscóides são de grande importância médica e sanitária por serem causadores de miíases secundárias e transmissores de microorganismos patogênicos como vírus, bactérias, cisto de protozoários e ovos de helmintos ao homem e animais domésticos (GREENBERG, 1971). Como o controle de moscas por inseticidas acaba selecionando populações resistentes, sendo um paliativo, Mendes \& Linhares (1993) acreditam necessária a pesquisa de novas metodologias visando ao controle de moscas.

Os adultos de Fannia pusio (Wiedemann, 1830) (Diptera: Fanniidae) são encontrados freqüentemente associados em ambientes modificados pelo homem, com suas larvas criando-se em matéria orgânica em decomposição (MARCHIORI \& PRADO, 1995, 1996; MARCHIORI et al., 2000a, b; PONT, 1977). Objetivou-se com este trabalho detectar as espécies de parasitóides de F. pusio coletadas em fezes humanas, fígado bovino e peixe, em Caldas Novas, Goiás, Brasil.

Como possibilidade de controle desses dípteros podem ser utilizados determinados grupos de parasitóides, agentes responsáveis pela redução de populações de algumas moscas (MARCHIORI et al., 2001).

'Professor Dr. Departamento de Agronomia do Instituto Luterano de Ensino Superior de Itumbiara, GO, ULBRA - Av. Beira Rio, 1001 - Bairro Nova Aurora - Itumbiara, GO - 75.500-000.

${ }^{2}$ Alunos do Departamento de Agronomia do Instituto Luterano de Ensino Superior de Itumbiara, GO, ULBRA - Av. Beira Rio, 1001 - Bairro Nova Aurora Itumbiara, GO - 75.500-000 - pesquisa.it@ulbra.br 
O estudo foi realizado no Parque da Serra de Caldas Novas localizada no município de Caldas Novas-GO $\left(17^{\circ} 44^{\prime} \mathrm{S}-48^{\circ} 37^{\prime} \mathrm{W}\right)$, quinzenalmente, no período de agosto de 2003 a maio de 2004. As moscas foram coletadas com o uso de armadilhas construídas com recipiente metálico (óleo de cozinha) de textura externa preta fosca, medindo cerca de $19 \mathrm{~cm}$ de altura por $9 \mathrm{~cm}$ de diâmetro, com duas aberturas tipo venezianas, confeccionadas no terço inferior para permitir a entrada dos insetos. Na parte superior da armadilha foi acoplado um funil de papel, aberto nas extremidades, com a base voltada para baixo e envolvido em um saco plástico, utilizado para a coleta das moscas e parasitóides atraídos. Utilizou-se como iscas: fezes humanas, fígado bovino e peixe depositados no interior dos recipientes metálicos, sobre uma camada de terra. Empregaram-se três armadilhas por tratamento, as quais foram penduradas em árvores a $1 \mathrm{~m}$ do solo e, distantes 2 m e uma das outras em área de cerrado. Os indivíduos adultos coletados foram levados para o laboratório, sacrificados com éter etílico e conservados em álcool $70 \%$ para identificação.

Tendo em vista uma eventual oviposição dos insetos atraídos, o conteúdo das armadilhas foi colocado em recipientes plásticos, contendo uma camada de areia para servir de substrato a pupação das larvas. Após 15 dias da colocação das armadilhas no campo, a areia foi peneirada e dela se recolheu as pupas, que em seguida foram individualizadas em cápsulas de gelatina (número 00) para obtenção de moscas e/ou parasitóides. A porcentagem de parasitismo foi calculada por meio do número de pupas parasitadas/número total de pupas obtidas x 100 . A identificação dos parasitóides foi realizada utilizando-se chave de identificação proposta por Díaz et al. (2000) e Legner et al. (1976), e a dos hospedeiros, baseou-se no trabalho de McAlpine et al. (1981). A preferência dos parasitóides pelo substrato onde foi coletada $F$. pusio foi testada pelo teste $X^{2}$, a $5,0 \%$ de significância.

Obtiveram-se 325 pupas de $F$. pusio, de 24 das quais emergiram 24 parasitóides, sendo 03 espécimens de Paraganaspis egeria Díaz, Gallardo \& Walsh, 1996 (Hymenoptera: Figitidae), 14 espécimens de Pachycrepoideus vindemmiae (Rondani, 1875) (Hymenoptera: Pteromalidae), 02 espécimens de Spalangia drosophilae Ashmead, 1887 (Hymenoptera: Pteromalidaee) e 05 espécimens de Spalangia nigra Latrielle, 1805 (Hymenoptera: Pteromalidae) (Tabela 1). A porcentagem de parasitismo obtida foi de $7,4 \%$

A porcentagem de parasitismo apresentada por $P$. vindemmiae, $S$. nigra, $P$. egeria, e $S$. drosophilae foi de $4,3,1,5,0,9$ e $0,6 \%$, respectivamente. A espécie de maior ocorrência foi $P$. vindemmiae representando 58,3\% dos indivíduos coletados e também aquela presente em todas as iscas atrativas (Tabela 1). Essa espécie é considerada um parasitóide solitário (quando uma única larva completa seu desenvolvimento em determinado hospedeiro) de dípteros das famílias Anthomyiidae, Calliphoridae, Muscidae, Sarcophagidae, Tachinidae e Tephritidae. Apresenta ampla distribuição geográfica sendo também encontrada na E.U.A. e África (HANSON \& GAULD, 1995).

TABELA 1 - Parasitóides de Fannia pusio (Wiedemann, 1830) coletados em três substratos, no município de Caldas Novas, Goiás, de agosto de 2003 a maio de 2004.

\begin{tabular}{|c|c|c|c|c|c|}
\hline Substrato & $\begin{array}{l}\text { Total de } \\
\text { pupas }\end{array}$ & Parasitóides & $\begin{array}{l}\text { Número de } \\
\text { indivíduos }\end{array}$ & $\begin{array}{l}\text { Número de } \\
\text { pupas } \\
\text { parasitadas }\end{array}$ & $\begin{array}{c}\text { Porcentagem } \\
\text { de parasitismo }\end{array}$ \\
\hline \multirow[t]{2}{*}{ Fezes humanas } & 237 & $\begin{array}{c}\text { Pachycrepoideus } \\
\text { vindemmiae }\end{array}$ & 10 & P & 4,2 \\
\hline & & Paraganaspis egeria & 03 & 03 & 1,3 \\
\hline \multirow[t]{3}{*}{ Fígado bovino } & 65 & $\begin{array}{c}\text { Pachycrepoideus } \\
\text { vindemmiae }\end{array}$ & 03 & 03 & 4,6 \\
\hline & & Spalangia drosophilae & 02 & 02 & 3,1 \\
\hline & & Spalangia nigra & 05 & 05 & 7,7 \\
\hline Peixe & 23 & $\begin{array}{c}\text { Pachycrepoideus } \\
\text { vindemmiae }\end{array}$ & 01 & 01 & 4,4 \\
\hline Total & 325 & & 24 & 24 & 7,4 \\
\hline
\end{tabular}


A espécie $S$. nigra foi a segunda mais abundante representando $20,8 \%$ dos indivíduos coletados e apresentou a maior freqüência de parasitismo com 7,7\%. Essa é uma espécie descrita originalmente da região Holártica com ampla distribuição E.U.A. e Canadá (LEGNER et al., 1976).

Com relação à preferência dos parasitóide pelos substratos verificou que: $P$. vindemmiae apresentou preferência por $F$. pusio coletada em fígado bovino; $P$. egeria ocorreu apenas em $F$. pusio coletada em fezes humanas; $S$. drosophilae e $S$ nigra também apresentaram preferências por $F$. pusio coletada em fígado bovino, contudo, foram coletadas apenas nesse substrato $\left(\mathrm{X}^{2}=14,78 ; \mathrm{GL}=6 ; \mathrm{P}<0,05\right)$.

Segundo Díaz \& Gallardo (1996), P. egeria provavelmente parasita larva de segundo estádio de seus hospedeiros. Essa espécie já foi encontrada parasitando Sarcophagula occidua (Fabricius, 1794) (Diptera: Sarcophagidae) (DÍAZ \& GALLARDO, 1996), Chrysomya albiceps (Wiedemann, 1805) (Diptera: Calliphoridae) (MARCHIORI, 2000) e Brontaea quadristigma (Thomson, 1869) (Diptera: Muscidae) (DÍAZ et al., 2000).

A espécie $S$. drosophilae é citada como parasitóide de pupas de dípteros pequenos das famílias Chloropidae, Drosophilidae, Muscidae, Sarcophagidae e Sepsidae (MARCHIORI, 2002; MARCHIORI et al., 2001, 2002).

\section{REFERÊNCIAS BIBLIOGRÁFICAS}

DÍAZ, N.; GALLARDO, F. Sobre cinipoideos del Brasil, parasitoides de dipteros estercoleros (Hymenoptera: Cynipoidea). Revista da Sociedad Entomologica Argentina, Buenos Aires, v. 55, n. 1-4, p. 127-129, 1996.

DÍAZ, N. B.; GALLARDO, F. E.; MARCHIORI, C. H. Cynipoidea parasitoids of dung-flies in Brazil: II (Insecta: Hymenoptera). Anais da Sociedade Entomológica do Brasil, Londrina, v. 29, n. 3, p. 469-474, 2000.

GREENBERG, B. Flies and disease: ecology, classification and biotic association. New Jersey: Princeton University, $1971.856 \mathrm{p}$.

HANSON, P. E.; GAULD, I. D. The Hymenoptera of Costa Rica. Oxford: Oxford University, 1995. 893 p.

LEGNER, E. E.; MOORE, I.; OLTON, G. S. Tabular keys \& biological notes to commom parasitoids of synanthropic
Diptera breeding in accumulated animal wastes. Entomological News, Philapdelphia, v. 87, p. 113-144, 1976.

MARCHIORI, C. H. Microhimenópteros parasitóides de moscas em esterco bovino em Cachoeira Dourada, Goiás, Brasil. Entomología Y Vectores, Rio de Janeiro, v. 9, p. 365374, 2002.

MARCHIORI, C. H. Parasitóides de estágios imaturos de dípteros sinantrópicos coletados em vários ambientes em Itumbiara-GO. Acta Scientiarum, Maringá, v. 22, p. 655$661,2000$.

MARCHIORI, C. H.; CALDAS, E. R.; DIAS, K. G. S. Parasitóides de Diptera em fezes bovinas em vários tempos de exposição em Itumbiara, Goiás, Brasil. Arquivos do Instituto Biológico, São Paulo, v. 69, p. 3742, 2002.

MARCHIORI, C. H.; CASTRO, M. E. V.; PAIVA, T. C. G.; SILVA, C. G.; TEIXEIRA, F. F. Dípteros muscóides de importância médica e veterinária e seus parasitóides em Goiás. Arquivo Brasileiro de Medicina Veterinária, Belo Horizonte, v. 52, p. 350-353, 2000a.

MARCHIORI, C. H.; OLIVEIRA, A. T.; LINHARES, A. X. Artrópodes associados a massas fecais bovinas no Sul do Estado de Goiás. Neotropical Entomology, Londrina, v. 30, p. 19-24, 2001.

MARCHIORI, C. H.; PRADO, A. P. Longevidade e fecundidade de Fannia pusio (Wiedemann, 1830) (Diptera: Fanniidae) em laboratório. Revista Brasileira de Biologia, São Carlos, v. 55, p. 571-575, 1995.

MARCHIORI, C. H.; PRADO, A. P. Efeito da temperatura no desenvolvimento dos estágios imaturos de Fannia pusio (Wiedemann, 1830) (Diptera: Fanniidae) em laboratório. Revista Brasileira de Biologia, São Carlos, v. 56, p. 93-98, 1996.

MARCHIORI, C. H.; SILVA, C. G.; CALDAS, E. R.; VIEIRA, C. I. S.; ALMEIDA, K. G. S.;TEIXEIRA, F. F.; LINHARES, A. X. Dípteros muscóides associados com carcaça de suíno e seus parasitóides em área de pastagem e de mata em Goiás. Arquivo Brasileiro de Medicina Veterinária e Zootecnia, Belo Horizonte, p. 459-460, 2000b. 
McALPINE, J. F.; PETERSON, B. V.; SHEWEELL, G. E.; espécies de Muscidae (Diptera). Revista Brasileira de TESKEY, H. J.; VOCKROTH, J. R.; WOOD, D. M. Manual of neartic Diptera. Otawa: Agriculture Canada, 1981. v. 2, 674 p.

Entomologia, Curitiba, v. 37, p. 289-297, 1993.

PONT, A. C. A revision of Australian Fanniidae (Diptera:

MENDES, J.; LINHARES, A. X. Atratividade por iscas, Calyptrate). Australian Journal of Zoology, Sidney, v. 1, n. sazonalidade e desenvolvimento ovariano em várias 51, p. 1-60, 1977. 